\title{
A Hybrid UCA-RARE/Root-MUSIC Approach for 2-D Direction of Arrival Estimation in Uniform Circular Arrays in the Presence of Mutual Coupling
}

\author{
Roald Goossens and Hendrik Rogier, Senior Member, IEEE
}

\begin{abstract}
A new hybrid algorithm that combines the uniform circular array-RAnk REduction (UCA-RARE) and Root-MUSIC algorithm for 2-D direction-of-arrival (DOA) estimation of azimuth and elevation angle is presented for uniform circular arrays in the presence of mutual coupling. To describe mutual coupling and platform effects we rely on the circular symmetry and expand the open-circuit voltages into a limited number of phase modes. This number of phase modes only depends on the electromagnetic dimensions of the UCA and is independent of the severity of mutual coupling in the UCA. The UCA-RARE algorithm is then applied to estimate the azimuth angle independent from the elevation angle. Next, for each azimuth angle we perform a new search-free rooting algorithm based on the expansion of the array manifold into a double Fourier series. By considering several examples, it is shown that even in the presence of severe mutual coupling the proposed combined technique yields very robust DOA estimations for azimuth angle as well as for elevation angle.
\end{abstract}

Index Terms-Antenna array, array signal processing, circular arrays, mobile communication, mutual coupling.

\section{INTRODUCTION}

$\mathbf{E}$ STIMATING the directions of arrival (DOAs) of plane waves impinging on an antenna array is an important issue in mobile communication systems. The knowledge of the DOAs can tremendously improve the performance of the mobile communication system. The symmetry present in a uniform circular array (UCA) makes this configuration attractive in the context of DOA estimation. In recent years several algorithms were proposed to estimate DOAs using UCAs, such as UCA-RB-MUSIC and UCA-ESPRIT [1]. However, the performance of these algorithms is often affected by mutual coupling between the antenna elements in the array. Especially in UCAs, mutual coupling can be significant. In [2]-[5] mutual coupling is compensated for by introducing a coupling matrix. The array manifold of the UCA, which is affected by mutual coupling, is projected by a coupling matrix onto the array manifold of an ideal UCA, where mutual coupling is not existing. In a general UCA configuration, however, the coupling matrix depends on the elevation angle [3]. This is especially so when platform effects, induced by scatterers in the near field [6], play an important role. For a fixed elevation

Manuscript received March 20, 2006; revised August 24, 2006.

The authors are with the Information Technology Department, Ghent University, Sint-Pietersnieuwstraat 41, Ghent, Belgium. (e-mail: roald. goossens@intec.UGent.be; hendrik.rogier@intec.UGent.be).

Color versions of one or more of the figures in this paper are available online at http://ieeexplore.ieee.org.

Digital Object Identifier 10.1109/TAP.2007.891848 angle it is still possible to construct a coupling matrix to estimate the azimuth angles of the impinging sources [7], provided that there are enough antenna elements in the UCA compared to its electrical dimensions. Yet, compensating for mutual coupling by a coupling matrix is not possible when there is no prior knowledge about the elevation angles, which is the case in this paper, because both azimuth and elevation angles are to be estimated. Besides algorithms which rely on the computation of the data covariance matrix, there is also a tendency to estimate the DOAs of the various signals by considering a single snapshot of the induced voltages [8], [9]. To apply the matrix pencil method, a method based on a single snapshot, a compensation of the mutual coupling by a coupling matrix is once more necessary to accurately estimate the DOAs. Another approach to compensate for mutual coupling is to use the presence of circular symmetry in the UCA. In spite of the presence of mutual coupling it is a reasonable assumption that the circular symmetry in the UCA is well preserved. In realistic UCAs the platform, the feed lines of the antenna elements and the wiring are the most important sources of mutual coupling. It is realistic that the geometry of the complete antenna system, including platform and feed lines, is carefully designed in order to preserve the circular symmetry. Due to the circular symmetry of the UCA the electromagnetic characteristics of all the antenna elements can be described by a limited number of parameters. In [7] it is proven that the open-circuit voltage of each element in the UCA can be described with a limited number of parameters by means of a spherical wave expansion. This approach makes it possible to easily adapt the MUSIC algorithm [10] in such manner that mutual coupling is compensated even in the presence of platform effects.

In this paper we estimate the DOAs in two dimensions, determining both the azimuth angle and elevation angle by making use of this spherical wave expansion. To perform a two-dimensional (2-D) estimation we need to ensure computational efficiency. To only estimate the azimuth angle, given a fixed elevation angle, efficient search-free algorithms (such as Root-MUSIC [1]), in which one can easily compensate for mutual coupling [11], have already been developed. The straightforward extension of MUSIC to a 2-D estimation of the DOA results in a 2-D search over the MUSIC spectrum, which is numerically inefficient. The UCA-ESPRIT algorithm [1] overcomes these problems by estimating the azimuth and elevation angles simultaneously. However, compensating for mutual coupling is difficult. To avoid an exhaustive 2-D search over the MUSIC spectrum we first perform the UCA-RAnk REduction (RARE) algorithm [12]. This eigenstructure-based 


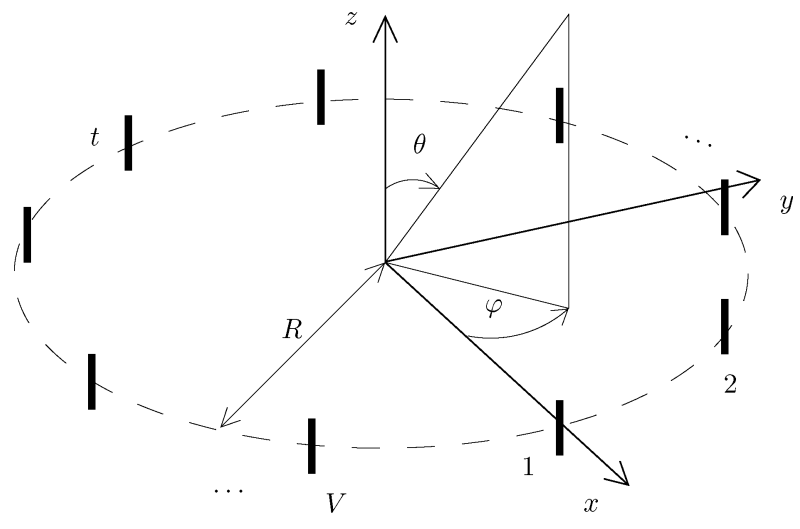

Fig. 1. Uniform circular array composed of $V$ antenna elements.

estimation method is developed to estimate the azimuth angles of the source directions decoupled from the elevation angles. We prove that this UCA-RARE algorithm can be applied even in the presence of severe mutual coupling. Although mutual coupling influences the electromagnetic characteristics, it is still possible to decompose the voltages induced over the antenna elements into a limited number of phase modes [7]. Together with the symmetry in the UCA, this is a sufficient condition as UCA-RARE only relies on these symmetry properties of the antenna array. In [13] we proved that UCA-RARE yields accurate estimates for the azimuth angle. The elevation angles were estimated by performing a one-dimensional (1-D) search over the MUSIC spectrum, which was achieved by an expansion of the array manifold into a limited number of spherical modes. This last search step is not numerically efficient so a new search-free rooting algorithm is proposed to estimate the elevation angles. In [14] it is proposed that wavefield modeling enables us to perform Root-MUSIC along any desired orientation angle. By a properly chosen extension of the array manifold we can rewrite the array manifold as a limited double Fourier series expansion. This allows the implementation of a Root-MUSIC algorithm in the elevation direction, which is numerically efficient.

In Section II we describe the electromagnetic characteristics of the UCA. Special attention is devoted to the situation where all antenna elements are terminated by the same load impedance. In Section III the UCA-RARE algorithm is presented, which delivers estimates for the azimuth angles independent from the elevation angles. The methods to estimate the elevation angles are explained in Section IV. In Section V some illustrative results are presented, proving that the proposed combined algorithm allows robust DOA estimation at low computational cost, delivering root-mean-square-errors (RMSEs) of the estimates that are close to their corresponding Cramer Rao bounds.

\section{ARray Signal Model}

Consider a uniform circular array (UCA) consisting of $V$ identical antenna elements (Fig. 1) distributed over a circle with radius $R$. The antenna elements operate at frequency $f$ with corresponding wave number $k$. We assume that antenna currents are only $z$-oriented. The phase center of each antenna element is located in the $x y$-plane, at azimuth angles $\varphi_{t}=(t-1) 2 \pi / V$ with

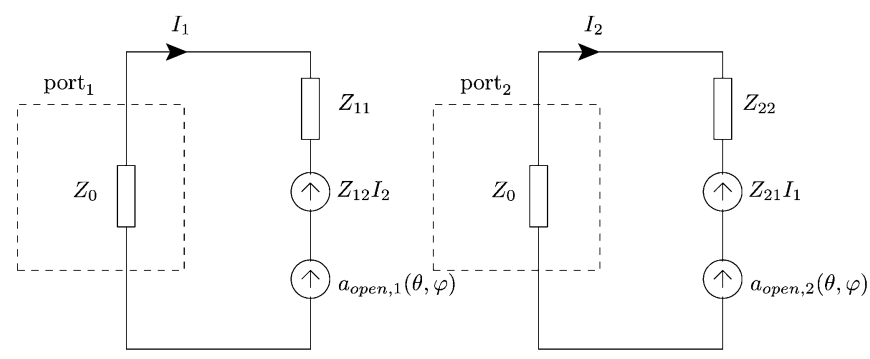

Fig. 2. Circuit models for a simple antenna array in receive mode.

$t=1,2, \ldots, V$. When $K$ plane waves impinge on the UCA the signal model of this array is given by

$$
\mathbf{x}(t)=\mathbf{A}(\boldsymbol{\theta}, \boldsymbol{\varphi}) \mathbf{s}(t)+\mathbf{n}(t)
$$

where $\mathbf{A}(\boldsymbol{\theta}, \boldsymbol{\varphi})(V \times K$ matrix $)$ is formed by the set of $K$ element space manifold vectors $\mathbf{a}\left(\theta_{k}, \varphi_{k}\right)(k=1,2, \ldots, K)$, determined by the DOAs $\left(\theta_{k}, \varphi_{k}\right)$ of the plane waves

$$
\mathbf{A}(\boldsymbol{\theta}, \boldsymbol{\varphi})=\left[\mathbf{a}\left(\theta_{1}, \varphi_{1}\right), \mathbf{a}\left(\theta_{2}, \varphi_{2}\right), \ldots, \mathbf{a}\left(\theta_{K}, \varphi_{K}\right)\right]
$$

$\mathbf{s}(t)$ is the $(K \times 1)$ signal vector and $\mathbf{n}(t)$ is the $(V \times 1)$ noise vector, where the noise is assumed to be additive white Gaussian noise. We restrict the signal model to UCAs which are composed of vertically polarized antennas, such as dipole antennas or vertically polarized aperture antennas. Only the vertically polarized components of incoming plane waves induce voltages over the loads of the UCA. The proposed combined algorithm is easily extendable to UCAs consisting of diversely polarized antenna elements, considering vertically polarized sources whose signals impinge on the antenna array. The knowledge of the element space manifold $\mathbf{a}(\theta, \varphi)$ of the uniform circular array

$$
\mathbf{a}(\theta, \varphi)=\left[a_{1}(\theta, \varphi), a_{2}(\theta, \varphi), \ldots, a_{V}(\theta, \varphi)\right]^{T}
$$

is essential in most algorithms for DOA estimation. The element space manifold $\mathbf{a}(\theta, \varphi)$ is the collection of voltages induced over the loads at the different antennas of the array when a plane wave, which is vertically polarized with a unit amplitude, impinges at the angles of arrival $(\theta, \varphi)$. We assume that all antennas are loaded by an identical load impedance $Z_{0}$. In absence of mutual coupling, the manifold element $a_{t}(\theta, \varphi)$ corresponds to the electromagnetic (EM) characteristics of the stand-alone antenna element $t$. Mutual coupling complicates the whole situation. In this case the voltages over the loads are given by

$$
\mathbf{a}(\theta, \varphi)=Z_{0}\left(\mathbf{Z}+Z_{0} \mathbf{I}\right)^{-1} \mathbf{a}_{\text {open }}(\theta, \varphi) .
$$

$\mathbf{a}_{\text {open }}(\theta, \varphi)$ is the collection of voltages induced at the antenna terminals of the array when all the antenna elements are open circuited. Because of the presence of the neighboring open-circuited elements, which induce shadowing effects, and nearby scatterers, which induce platform effects, these voltages differ from the voltages in the stand-alone case [6]. In Fig. 2, the circuit model for a simple antenna array in receive mode is shown. Clearly, the system is completely characterized by the 
impedance matrix $\mathbf{Z}$ and the vector $\mathbf{a}_{\text {open }}(\theta, \varphi)$, containing the open-circuit voltage sources. The impedance matrix $\mathbf{Z}$ can easily be found by exciting antenna $t(t=1,2, \ldots, V)$ with a $1 A$ current source and measuring the voltages at the other antennas which are open circuited. The measured voltages are $[\mathrm{Z}]_{t k} .1 A(k=1 \ldots V)$. The open-circuit voltages are found in a similar fashion. When applying the excitation scheme defined above, the radiation pattern $\mathbf{F}_{t}(\theta, \varphi)$ of antenna $t$ is directly related to the open-circuit voltage of antenna $t$ in receive mode through [15], [16]

$$
a_{\text {open,t }}(\theta, \varphi)=-\frac{2 j \lambda}{R_{c}} \frac{\mathrm{e}^{\mathrm{inc}}(\theta, \varphi) \cdot \mathbf{F}_{t}(\theta, \varphi)}{1 A} .
$$

$\mathrm{e}^{\text {inc }}(\theta, \varphi)$ represents an incoming plane wave at angles $(\theta, \varphi)$, where $\lambda$ is the wavelength and $R_{c}$ the impedance of the medium in which the antenna radiates. One of the advantages of uniform circular arrays is the existence of a high degree of symmetry (we assume that mutual coupling, shadowing and platform effects are such that the circular symmetry is maintained). In a UCA, the following relationship exists between the radiation patterns/ open-circuit voltages of the different antenna elements

$$
\left\{\begin{array}{l}
\mathbf{F}_{t}(\theta, \varphi)=\mathbf{F}_{1}\left(\theta, \varphi-\varphi_{t}\right) \\
a_{\text {open }, t}(\theta, \varphi)=a_{\text {open }, 1}\left(\theta, \varphi-\varphi_{t}\right)
\end{array} .\right.
$$

Eventually, the array signal model can be described in full electromagnetic detail by a single column of the impedance matrix $\mathbf{Z}$ and the open-circuit voltage $a_{\text {open, } 1}(\theta, \varphi)$. Notice that we opt for describing mutual coupling by measuring the far-fields emitted by the different antenna elements. Mutual coupling can also be described by considering the detailed current distribution on all the antennas and nearly scatterers. However, our description is more compact, since all near field information has been removed. Yet the description remains rigorous in describing the radiated fields (transmit mode of the antenna array) or in describing the voltages induced by an incident plane wave (receive mode of the antenna array).

In order to implement numerical efficient DOA estimation algorithms we describe the electromagnetic characteristics, i.e., the array manifold, by a limited number of parameters. In [1], eigenstructure techniques with UCAs are proposed in absence of mutual coupling. The UCAs are composed of omnidirectional antenna elements, so the voltages induced over the antennas depend only on the position of the elements (no mutual coupling is considered)

$$
\begin{aligned}
a_{\mathrm{omni}, t}(\theta, \varphi) & =e^{j k R \sin \theta \cos \left(\varphi-\varphi_{t}\right)} \\
& =\sum_{m=-\infty}^{+\infty} j^{m} J_{m}(k R \sin \theta) e^{-j m \varphi_{t}} e^{j m \varphi}
\end{aligned}
$$

where $J_{m}(k R \sin \theta)$ is the Bessel function of the first kind of order $m$. The mode amplitude $J_{m}(k R \sin \theta)$ is small when the Bessel function order $m$ exceeds its argument $k R \sin \theta$. So the summation can be truncated from $-M$ to $+M$, where $M \approx k R$. In combination with a sufficiently large number of antenna elements a phase mode excitation-based beamforming can be realized. The structure of the array manifold, after the beamspace operation, is the basis for the development of algorithms such as UCA-RB-MUSIC [1] and UCA-RARE [12]. In [7] it is shown that the open-circuit voltage of an antenna in the UCA, as considered in this paper, can be described in a similar way:

$$
a_{\text {open }, t}(\theta, \varphi)=\sin \theta \sum_{m=-M}^{+M} V_{m}^{o}(\theta) e^{-j m \varphi_{t}} e^{j m \varphi} .
$$

The Bessel function of the first kind of order $m$ is replaced by $\sin \theta V_{m}^{o}(\theta)$ and these coefficients, which are obtained by measuring the radiation pattern $\mathbf{F}_{t}(\theta, \varphi)$, describe the electromagnetic characteristics of the UCA, including mutual coupling. The electromagnetic dimensions of the UCA restrict the number of terms in the summation (8) to $2 M+1$, where $M>k R$.

Given (4) and given that $\mathbf{Z}$ is a circulant matrix, because of the circular symmetry of the array, we can derive similar properties for the element space manifold $\mathbf{a}(\theta, \varphi)$ of the loaded voltages as for the manifold $\mathbf{a}_{\text {open }}(\theta, \varphi)$ of the open-circuit voltages

$$
\begin{aligned}
& a_{t}(\theta, \varphi)=a_{1}\left(\theta, \varphi-\varphi_{t}\right) \\
& a_{t}(\theta, \varphi)=\sin \theta \sum_{m=-M}^{+M} V_{m}^{l}(\theta) e^{-j m \varphi_{t}} e^{j m \varphi} .
\end{aligned}
$$

There exists a simple relation between $V_{m}^{o}(\theta)$ and $V_{m}^{l}(\theta)$ [7]

$$
\begin{aligned}
V_{m}^{l}(\theta) & =\frac{Z_{0}}{Z_{m}^{\phi}+Z_{0}} V_{m}^{o}(\theta) \\
Z_{m}^{\phi} & =\sum_{n=1}^{V}[\mathbf{Z}]_{1, n} e^{-j m 2 \pi(1-n) / V} .
\end{aligned}
$$

With this description of the element array manifold we now proceed to apply UCA-RARE on the UCA in the presence of mutual coupling.

\section{UCA-RARE}

The MUSIC eigenstructure technique [10] can be easily adapted to account for mutual coupling. Only the knowledge of the array manifold, affected by mutual coupling, is necessary to perform DOA estimation based on the MUSIC algorithm in the presence of mutual coupling. Yet it requires an extensive 2-D search to obtain estimates for both azimuth and elevation angles. The introduction of a mutual coupling matrix [2]-[5] which maps the array manifold of a UCA with mutual coupling onto the array manifold of an ideal UCA, is not applicable in the situation where severe mutual coupling effects exist, such as platform effects (in Section V examples will be given). In this case the coupling matrix (notice that this matrix differs from the impedance matrix $\mathbf{Z}$ defined in this paper) depends on the elevation angle. In this paper we simultaneously estimate azimuth angle as well as elevation angle, so no prior knowledge about the elevation angle is assumed. Nevertheless, we avoid the computationally expensive 2-D search by applying the UCA-RARE algorithm. This algorithm estimates the azimuth angles of the impinging sources without prior knowledge of the elevation angles. This algorithm was developed by Pesavento and Böhme [12] for the case of unknown directional patterns. In [12] no mutual coupling effects were considered. Mutual coupling in the UCA deforms the directional patterns, yet the symmetry property (9) still holds. As UCA-RARE only relies 
on this symmetry property and not on the detailed knowledge of the antenna patterns, it can be used in array configurations in which mutual coupling effects are significant.

We start from the data model of Section II. $K$ plane waves impinge on the UCA. First of all the data undergo an FFT (Fast Fourier Transform). This beamspace transformation is a first step in a well-established method for DOA estimation in UCAs [1]. It transforms the array manifold into a beamspace manifold, which is more convenient for rooting algorithms that estimate the DOAs

$$
\begin{aligned}
\mathbf{x}_{\text {beam }}(t)= & \mathbf{W} \mathbf{x}(t) \\
{[\mathbf{W}]_{s, t}=} & \frac{1}{\sqrt{V}} e^{j 2 \pi / V(s-M-1)(t-1)} \\
& \text { with } t=1 \ldots V \text { and } s=1 \ldots 2 M+1 .
\end{aligned}
$$

$M$ is the same as in the expansion of the open-circuit voltages (8) and only depends on the dimensions of the array. The covariance matrix $\mathbf{R}$ of the beamspace data is constructed and an eigendecomposition of $\mathbf{R}$ results in a signal and noise subspace

$$
\mathbf{R}=E\left\{\mathbf{x}_{\text {beam }}(t) \mathbf{x}_{\text {beam }}(t)^{H}\right\}=\mathbf{E}_{S} \boldsymbol{\Lambda}_{S} \mathbf{E}_{S}^{H}+\mathbf{E}_{N} \boldsymbol{\Lambda}_{N} \mathbf{E}_{N}^{H} .
$$

The diagonal matrices $\Lambda_{N} \in \mathbb{R}^{(2 M+1-K) \times(2 M+1-K)}$ and $\Lambda_{S} \in \mathbb{R}^{K \times K}$ contain the noise subspace and signal subspace eigenvalues of $\mathbf{R}$, respectively. In turn, the columns of the matrices $\mathbf{E}_{N} \in \mathbb{C}^{(2 M+1) \times(2 M+1-K)}$ and $\mathbf{E}_{S} \in \mathbb{C}^{(2 M+1) \times K}$ denote the corresponding noise subspace and signal subspace eigenvectors. The beamspace MUSIC algorithm estimates the signal DOAs $(\theta, \varphi)$ from the $K$ deepest nulls of the MUSIC function, which is the projection of the beamspace array manifold onto the noise subspace

$$
f_{\text {MUSIC }}(\theta, \varphi)=\mathbf{a}_{\text {beam }}^{H}(\theta, \varphi) \mathbf{E}_{N} \mathbf{E}_{N}^{H} \mathbf{a}_{\text {beam }}(\theta, \varphi)
$$

with the beamspace manifold $\mathbf{a}_{\text {beam }}(\theta, \varphi)=\mathbf{W} \mathbf{a}(\theta, \varphi)$. When the number of antenna elements $V$ is sufficiently high, i.e., $V \geq$ $2 M+1$, the beamspace transformation (13) will excite one single phase mode such that

$$
\begin{aligned}
\sum_{t=1}^{V}[\mathbf{W}]_{m+M+1, t} a_{t}(\theta, \varphi)= & \sin \theta V_{m}^{l}(\theta) e^{j m \varphi} \\
& \text { with } m=-M, \ldots,+M .
\end{aligned}
$$

In combination with the symmetry property, $V_{-m}^{l}(\theta)=V_{m}^{l}(\theta)$, which is generally valid for UCAs consisting of vertically polarized antennas (this implies a symmetric antenna pattern, e.g., $\mathbf{F}(\theta,-\varphi)=\mathbf{F}(\theta, \varphi))$, the factorization of the beamspace manifold is achieved

$$
\begin{aligned}
\mathbf{a}_{\text {beam }}(\theta, \varphi) & =\mathbf{T}\left(e^{j \varphi}\right) \mathbf{g}(\theta), \\
{[\mathrm{g}(\theta)]_{k} } & =\sin \theta V_{M-k+1}^{l}(\theta) \quad k=1 \ldots M+1 \\
\mathbf{T}(z) & =\left[\begin{array}{cc}
\mathbf{Q}(z) & 0 \\
0 & 1 \\
\Pi \mathbf{Q}\left(\frac{1}{z}\right) & 0
\end{array}\right] \\
\mathbf{Q}(z) & =\operatorname{diag}\left\{z^{-M}, z^{-M+1}, \ldots, z^{-2}, z^{-1}\right\}
\end{aligned}
$$

with

$$
z=e^{j \varphi}
$$

where $\Pi$ is the $M \times M$ anti-diagonal unit matrix. In spite of the presence of mutual coupling the beamspace manifold can still be factorized into a function of the azimuth angle $\varphi$ and the elevation angle $\theta$, in the same manner as in [12].

Notice that in absence of mutual coupling and using omnidirectional antenna elements $V_{m}^{l}(\theta)$ is replaced by $j^{m} J(k r \sin \theta)$, the Bessel function of the first kind of order $m$. Replacing the beamspace manifold by its factorization (18), the MUSIC function becomes

$$
f_{\text {MUSIC }}(\theta, \varphi)=\mathbf{g}^{H}(\theta) \mathbf{T}^{H}(z) \mathbf{E}_{N} \mathbf{E}_{N}^{H} \mathbf{T}(z) \mathbf{g}(\theta) .
$$

If the source signals impinge on the UCA at a known elevation angle, the MUSIC function (22) can be written as a polynomial in $z=e^{j \varphi}$ and a Root-MUSIC algorithm can deliver estimates for the azimuth angles [1]. However, no prior information about the elevation angle is available. The factorization of the beamspace manifold enables us to perform a rank reduction algorithm, namely UCA-RARE. The minimization problem (22) is relaxed by extending the set of possible solutions in the original manifold $\left\{\mathbf{a}_{\text {beam }}(\theta, \varphi),(\theta, \varphi) \in \Omega\right\}$ to a larger set of possible solutions in the so-called RARE manifold $\left\{\mathbf{T}\left(e^{j \varphi}\right) \mathbf{c}, \varphi \in[0,2 \pi], \mathbf{c} \in \mathbb{C}^{2 M+1 \times 1}\right\} . \mathbf{c}$ is an arbitrary complex vector. Equation (22) becomes

$$
f_{\mathrm{MUSIC}}(\theta, \varphi)=\mathbf{c}^{H} \mathbf{T}^{H}(z) \mathbf{E}_{N} \mathbf{E}_{N}^{H} \mathbf{T}(z) \mathbf{c}=0 .
$$

Estimates for the azimuth angles are obtained, independent of the elevation angle, by searching for the nulls of (23). This can be reduced to the 1-D polynomial criterion

$$
P_{\mathrm{RARE}}(z)_{|z|=1}=\operatorname{det}\left\{\mathbf{T}\left(\frac{1}{z}\right)^{T} \mathbf{E}_{N} \mathbf{E}_{N}^{H} \mathbf{T}(z)\right\}=0 .
$$

The study of the larger set of the manifold shows that spurious states might be introduced. In [12] one proves that the spurious states also satisfy

$$
P_{\mathrm{SPUR}}(z)_{|z|=1}=\operatorname{det}\left\{\mathbf{E}_{S}^{H} \mathbf{T}(z) \Delta \mathbf{T}\left(\frac{1}{z}\right)^{T} \mathbf{E}_{S}\right\}=0
$$

with $\boldsymbol{\Delta}=\left(\mathbf{T}(1 / z)^{T} \mathbf{T}(z)\right)^{-1}$. Estimates of $\varphi$ which are a solution of both (24) and (25) are rejected.

Notice that the azimuth estimates, a result of the UCA-RARE algorithm, satisfy the property that $\varphi$ as well as the anti-supplementary angle $\pi+\varphi$ are possible estimates. The appearance of these pairs of estimates is used in Section IV where a new algorithm to estimate the corresponding elevation angle is discussed.

\section{ROOT-MUSIC IN ELEVATION DIRECTION}

Through polynomial rooting, the UCA-RARE algorithm provides estimates of the azimuth angles $\left\{\left(\widehat{\varphi}_{1}, \widehat{\varphi}_{1}+\pi\right),\left(\widehat{\varphi}_{2}, \widehat{\varphi}_{2}+\right.\right.$ $\left.\pi), \ldots,\left(\widehat{\varphi}_{q}, \widehat{\varphi}_{q}+\pi\right)\right\}$ without requiring any information about the elevation angle. The estimates appear in pairs and we assume that the UCA-RARE algorithm yields $q$ pairs of azimuth angles. However, given that $\mathrm{K}$ sources impinge on the array, we do not consider that $q=K$. It is possible that several signals hit the UCA along the same azimuth angle and so this can reduce the value of $q$. In the presence of strong noise (low SNR) it is not certain that spurious states, which are introduced by 
the relaxation of the search procedure in Section III, still satisfy the condition (25). This results in an increase of the value $q$. In this section we describe how estimates for the elevation angles are obtained on the basis of the paired estimates of the azimuth angle.

In [14] it is reported that wavefield modeling enables us to perform a Root-MUSIC algorithm along any desired orientation angle. We apply this principle by performing a Root-MUSIC algorithm to estimate the elevation angle $\theta$ for every estimate of the azimuth angle, which is obtained by UCA-RARE. For that purpose we extend the element array manifold in such a manner that the element manifold is defined for values of $\theta$ in $[0,2 \pi]$

$$
b_{t}(\theta, \varphi)=\left\{\begin{array}{l}
a_{t}(\theta, \varphi) \text { for } \varphi=[0,2 \pi], \theta=[0, \pi] \\
-a_{t}(2 \pi-\theta, \pi+\varphi) \text { for } \varphi=[0,2 \pi], \theta=[\pi, 2 \pi] .
\end{array}\right.
$$

In the Appendix, we prove that it is possible to apply a double Fourier transform on the extended element manifold

$$
\begin{aligned}
b_{t}(\theta, \varphi) & =\sum_{m=-M}^{M} \sum_{n=-N}^{N} f_{m n} e^{j m\left(\varphi-\varphi_{t}\right)} e^{j n \theta} \\
& =\sum_{m=-M}^{M} \sum_{n=-N}^{N} f_{m n} z^{m} w^{n} e^{-j m \varphi_{t}} .
\end{aligned}
$$

In both dimensions the summation is restricted to a finite number of terms $M$ and $N$, which are bounded by the electromagnetic dimensions of the UCA

$$
\begin{aligned}
M & >k R \\
N & >k \sqrt{R^{2}+z_{\max }^{2}}+1
\end{aligned}
$$

where $z_{\max }$ is the maximal dimension of the UCA in $z$-direction. This expansion describes the electromagnetic characteristics of the UCA in full detail, because it is based on the knowledge of the impedance matrix $\mathbf{Z}$ and the open-circuit voltage $a_{\text {open }, t}(\theta, \varphi)$. The coefficients of this double Fourier expansion $\left\{f_{m n}\right\}$ are further in this paper denoted as the $f$-coefficients of the UCA. The element manifold can be written as a multivariate polynomial in $z=e^{j \varphi}$ and $w=e^{j \theta}$. This property opens up new possibilities to estimate the elevation angle in a computationally efficient manner. We perform a beamspace operation (13) to the extended element manifold

$$
\mathbf{b}_{\text {beam }}(\theta, \varphi)=\mathbf{W} \mathbf{b}(\theta, \varphi)=\Gamma(\varphi) \mathbf{F} \mathbf{w}
$$

with

$$
\begin{aligned}
& \Gamma(\varphi)=\left[\begin{array}{cccc}
z^{-M} & & & \\
& \cdot & & \\
& \cdot & \\
& & \cdot & \\
& & z^{M}
\end{array}\right] \\
& \text { with } \quad z=e^{j \varphi}, \\
& {[\mathbf{F}]_{m+M+1, n+N+1}=} f_{m n}, \quad m=-M \cdots+M \\
& n=-N-1 \ldots N+1
\end{aligned}
$$

and

$$
[\mathbf{w}]_{n+N+1}=e^{j n \theta}=w^{n} \quad n=-N \ldots N .
$$

The beamspace MUSIC function becomes

$$
\begin{aligned}
f_{\text {MUSIC }}(\theta, \varphi) & =\mathbf{b}_{\text {beam }}(\theta, \varphi)^{H} \mathbf{E}_{N} \mathbf{E}_{N}^{H} \mathbf{b}_{\text {beam }}(\theta, \varphi) \\
& =\mathbf{w}^{H} \mathbf{F}^{H} \boldsymbol{\Gamma}^{H}(\varphi) \mathbf{E}_{N} \mathbf{E}_{N}^{H} \boldsymbol{\Gamma}(\varphi) \mathbf{F} \mathbf{w} \\
& =\mathbf{w}^{H} \mathbf{Q} \mathbf{Q}^{H} \mathbf{w} .
\end{aligned}
$$

The noise subspace $\mathbf{E}_{N}$ in (34) is the same as the noise subspace used in the UCA-RARE algorithm (15). Given a paired estimate of the azimuth angle $\left\{\widehat{\varphi}_{i}, \widehat{\varphi}_{i}+\pi\right\}$ we evaluate $\mathbf{Q}=$ $\mathbf{F}^{H} \boldsymbol{\Gamma}^{H}\left(\widehat{\varphi}_{i}\right) \mathbf{E}_{N}$ and perform a rooting algorithm over the elevation angle $\theta$. The phases of the complex zeros of (34), that lie the closest to the unit circle, serve as estimates for the elevation angle $\left\{\widehat{\theta}_{s}^{i}\right\}$ with $s=1 \ldots S_{i}$. Depending on the value of $\widehat{\theta}_{s}^{i}$, we pair these estimates to $\widehat{\varphi}_{i}$ or $\widehat{\varphi}_{i}+\pi$

$$
\left\{\begin{array}{l}
\widehat{\theta}_{s}^{i} \in[0, \pi] \Longrightarrow\left(\widehat{\theta}_{s}^{i}, \widehat{\varphi}_{i}\right) \\
\widehat{\theta}_{s}^{i} \in[\pi, 2 \pi] \Longrightarrow\left(2 \pi-\widehat{\theta}_{s}^{i}, \widehat{\varphi}_{i}+\pi\right) .
\end{array}\right.
$$

Notice that the UCA-RARE algorithm delivers $2 q$ estimates for the azimuth angle ( $q$ paired estimates). By a properly chosen extension of the array manifold only $q$ implementations of the Root-MUSIC algorithm in elevation angle have to be performed, because in one implementation the nulls are searched in elevation direction for an azimuth angle and its anti-supplementary angle.

Also in this procedure spurious states might be introduced, so that some nulls obtained in the Root-MUSIC algorithm will not correspond to a realistic DOA. To solve this problem we calculate the MUSIC function for every paired estimate $\left(\widehat{\theta}_{s}^{i}, \widehat{\varphi}_{i}\right)$ ( $i=1 \ldots q$ and $\left.s=1 \ldots S_{i}\right)$. Only the $K$ smallest values of the MUSIC function are considered as final estimates for the DOAs $(\theta, \varphi)$.

\section{RESULTS}

Consider a uniform circular array consisting of nine dipole antenna elements tuned to $900 \mathrm{MHz}$ (dipole length $l=16.12 \mathrm{~cm})$. The array elements are distributed uniformly on a circle with diameter $d=l(\approx \lambda / 2)$. In the center of the circle there is a short-circuited dipole with length $l=16.12 \mathrm{~cm}$. All numerical results given in this section pertain to this array configuration. The short-circuited dipole acts as a platform effect and compensation of mutual coupling by means of a coupling matrix is not straightforward. This is illustrated in Fig. 3, where the open-circuit voltages of an antenna element in different array configurations are shown. In the UCA without central short-circuited dipole the open-circuit voltage of an array element is slightly different from the open-circuit voltage of a stand-alone dipole element. In presence of the center element the open-circuit voltage is much more dependent on the azimuth angle of the incoming plane wave. Yet, it is still possible to introduce a coupling matrix that maps the array manifold of the UCA, which is affected by platform effects, onto the array manifold of an ideal UCA [7]. Yet this mutual 


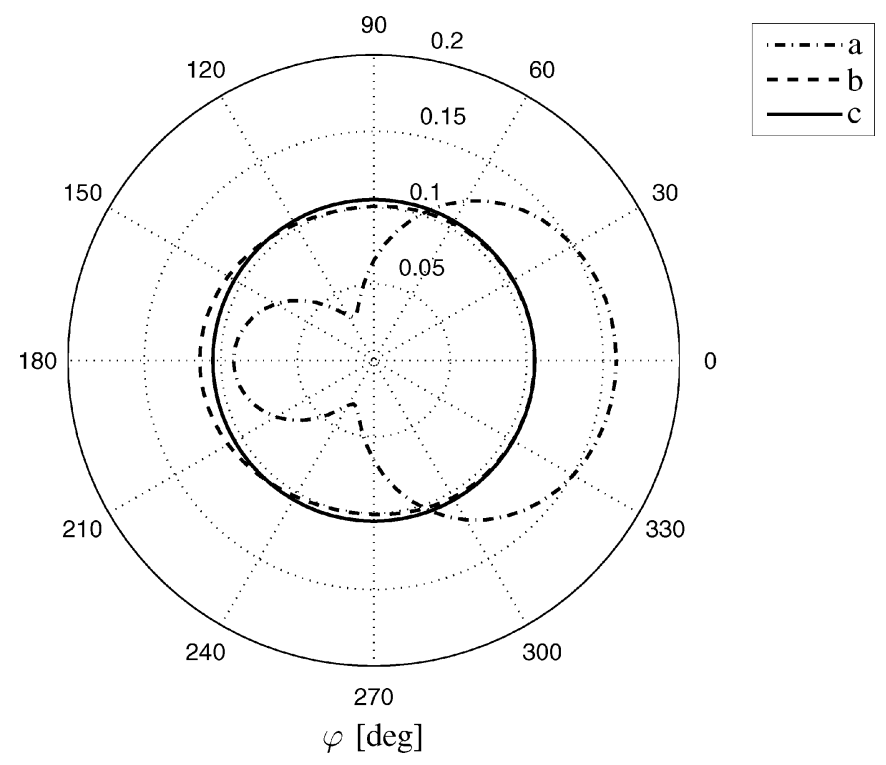

Fig. 3. Open-circuit voltage of an antenna element at $\theta=90^{\circ}$ for a: UCA with short-circuited dipole, b: UCA without short-circuited dipole, c: stand-alone dipole.

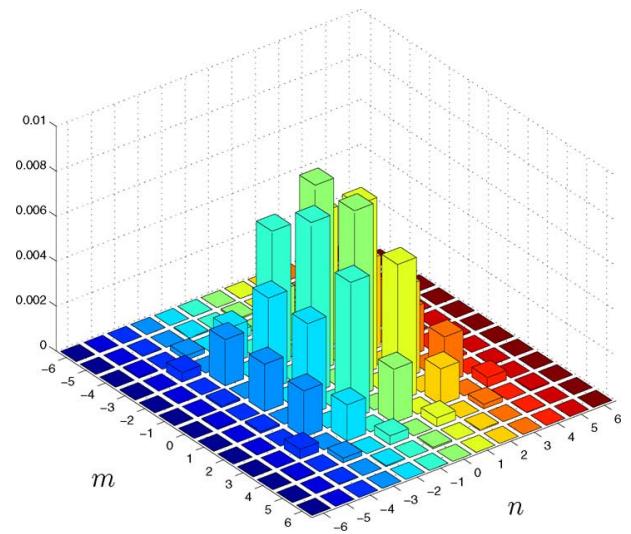

Fig. 4. The $f$-coefficients $\left|f_{m n}\right|$ for the nine-element array at $900 \mathrm{MHz}$.

coupling matrix depends on the elevation angle $\theta$ because of the platform effects. To obtain accurate estimates of the DOAs in two dimensions it is necessary to rely on the double Fourier expansion proposed in (27). Mutual coupling and generally the global electromagnetic characteristics of the UCA, including the platform effects, are described by the $f$-coefficients. In Fig. 4 the $f$-coefficients are plotted for the UCA under study. Only a limited number of coefficients is necessary to describe the induced voltages over the antenna elements. $f_{m n}$ is sufficient small when $m>4$ and $n>4$, so $M=4$ and $N=4$. This complies with the conditions (28) and (29): $M=4>k R=1.6$ and $N=4>k \sqrt{R^{2}+z_{\max }^{2}}+1=3.2$. To perform UCA-RARE, the number of antennas has to be sufficiently large $(V \geq 2 M+1)$. Hence, nine antenna elements are sufficient to perform a beamspace operation which results in a factorization following (18).

Now all antenna elements are loaded with a termination $Z_{0}=73 \Omega$. To demonstrate the new algorithm proposed in this paper, consider three uncorrelated sources emitting 100 bit pseudo-random bit sequences. The DOAs of the three signals are $\left(\theta_{1}, \varphi_{1}\right)=\left(60^{\circ}, 20^{\circ}\right),\left(\theta_{2}, \varphi_{2}\right)=\left(70^{\circ}, 200^{\circ}\right)$ and $\left(\theta_{3}, \varphi_{3}\right)=\left(55^{\circ}, 285^{\circ}\right)$. All sources impinge on the array from the upper hemisphere. This restriction counteracts the ambiguity in the elevation angle. The symmetry in the considered UCA results in the fact that the DOAs $(\theta, \varphi)$ and $(\pi-\theta, \varphi)$ cannot be distinguished because sources from both DOAs result in the same voltages induced over the antenna elements. UCAs which are not symmetric in elevation angle do not suffer from this ambiguity. The signals are received in the presence of additive white Gaussian noise. We estimate the azimuth and elevation angle by the combined algorithm proposed in Sections III and IV. All results are averaged over 1000 ensembles and the RMSE is calculated. These calculations are repeated for different SNR levels.

Prudence is called for the definition of the SNR level. Generally omnidirectional antenna elements are used in DOA estimation algorithms. In this paper more realistic antenna configurations are considered. In the case of omnidirectional elements the situation is clear, the SNR level is defined by

$$
\mathrm{SNR}=\frac{E\left[\mathbf{a}_{\mathrm{omni}}(\theta, \varphi)^{H} \mathbf{a}_{\mathrm{omni}}(\theta, \varphi) s^{*}(t) s(t)\right]}{E\left[\mathbf{n}(t)^{H} \mathbf{n}(t)\right]}=\frac{V P}{V \sigma^{2}}
$$

where $P=E\left[s^{*}(t) s(t)\right]$ is the source variance, $\sigma^{2}=$ $E\left[n_{i}^{*}(t) n_{i}(t)\right]$ the noise variance and $V$ the number of antenna elements. There is no angular dependency as aspected. In case of our realistic UCA, the SNR level is defined by

$$
\mathrm{SNR}=\frac{E\left[\mathbf{a}(\theta, \varphi)^{H} \mathbf{a}(\theta, \varphi) s^{*}(t) s(t)\right]}{E\left[\mathbf{n}(t)^{H} \mathbf{n}(t)\right]}=\frac{g(\theta) P}{V \sigma^{2}} .
$$

Because of the circular symmetry in the array there is a dependency in the elevation angle only. In Fig. $6 g(\theta)$ is shown for the UCA with the short-circuited element in the center. Because of the vertical dipole elements in the UCA we notice that for elevation angles near the poles $\theta=0^{\circ}$ and $\theta=180^{\circ}$ signals are hardly received and the SNR level will tend to zero, while for the elevation angle $\theta=90^{\circ}$ there is a maximum receive capacity. When several signals hit the UCA, one signal is chosen as reference signal to define the SNR level. In our first example we chose the third signal as a reference signal. We assume also that all signals have an equal source variance $E\left[s_{1}^{*}(t) s_{1}(t)\right]=E\left[s_{2}^{*}(t) s_{2}(t)\right]=E\left[s_{3}^{*}(t) s_{3}(t)\right]=P$. This assumption does not affect the generality of the results. Yet, due to this assumption the electromagnetic characteristics of the UCA are well illustrated. Depending on the elevation angle, the signal will be received properly and this results in a lower or higher SNR level. A lower SNR level is obtained than the reference SNR level when the signal reception is worse in comparison with the reference signal. In the first example the SNR levels are defined by

$$
\begin{aligned}
& \mathrm{SNR}_{1}=\mathrm{SNR}_{3} \frac{g\left(\theta_{1}\right)}{g\left(\theta_{3}\right)}=1.64 \mathrm{SNR} \\
& \mathrm{SNR}_{2}=\mathrm{SNR}_{3} \frac{g\left(\theta_{2}\right)}{g\left(\theta_{3}\right)}=2.36 \mathrm{SNR} \\
& \mathrm{SNR}_{3}=\mathrm{SNR}
\end{aligned}
$$

Expressed in decibels the equations become

$$
\begin{aligned}
& \mathrm{SNR}_{1}(\mathrm{~dB})=\mathrm{SNR}(\mathrm{dB})+4.30 \mathrm{~dB} \\
& \mathrm{SNR}_{2}(\mathrm{~dB})=\operatorname{SNR}(\mathrm{dB})+7.45 \mathrm{~dB} .
\end{aligned}
$$



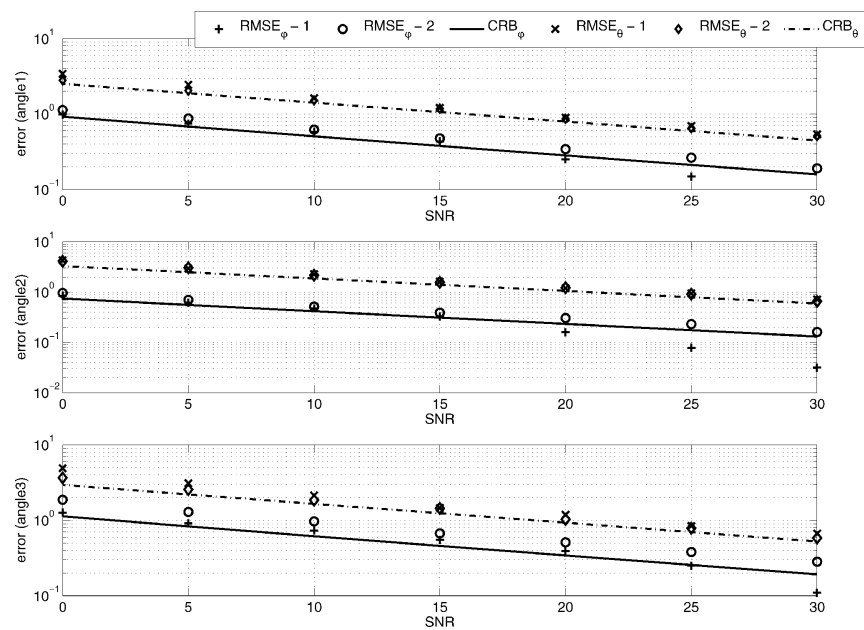

Fig. 5. RMSEs [deg] obtained by method 1 (2-D MUSIC search) and method 2 (UCA-RARE + Root-MUSIC) and corresponding CRBs of azimuth and elevation angles for the 3 different sources versus $\mathrm{SNR}$ level $\left(\mathrm{SNR}_{3}\right)$.

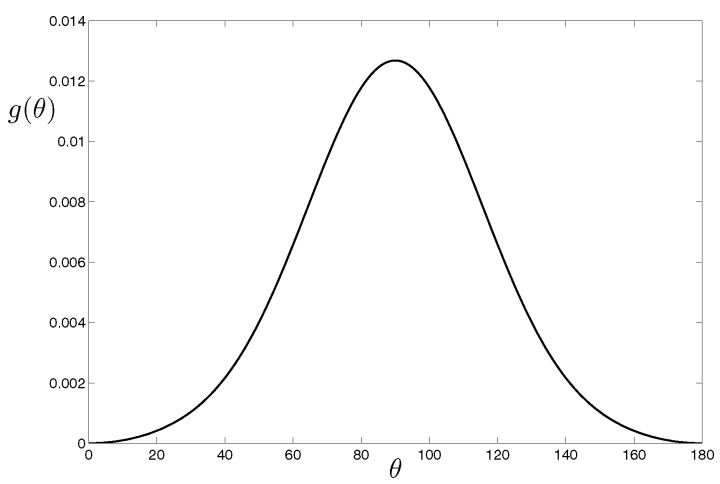

Fig. 6. $g(\theta)$ for the considered UCA.

In Fig. 5 the RMSEs obtained by the combined algorithm are compared to the RMSEs obtained by means of a well-known technique, a search over the 2-D MUSIC spectrum. This technique is the most straightforward technique to estimate the DOAs. The knowledge of the array manifold, e.g., obtained by measurements or by simulations to incorporate all mutual coupling effects, enables us to calculate the 2-D MUSIC spectrum. Obviously searching the highest local peaks in this spectrum yields the DOA estimates. In our case the MUSIC spectrum is calculated for every degree in elevation and azimuth direction, obtaining a $360 \times 181$ matrix. In Fig. 5 we see that both techniques have almost the same estimation accuracy. Nevertheless the computational efficiency differs for both techniques. The average MatLab-runtime to estimate the DOAs by the combined algorithm is $0.11 \mathrm{~s}$ (simulated on a $2.4 \mathrm{GHz}$ Pentium $I V$ Processor), whereas the average MatLab-runtime by the traditional search of the 2-D MUSIC spectrum is $1.87 \mathrm{~s}$. This reduction of the Matlab-runtime can be attributed to the two computational efficient rooting algorithms (UCA-RARE and the modified Root-MUSIC algorithm) which replace the 2-D search in the traditional 2-D MUSIC algorithm. In Fig. 5 the corresponding Cramer Rao bounds (CRB) are also shown. The CRB defines the ultimate accuracy of any estimation procedure in the presence of noise. It is clear that the RMSE obtained by

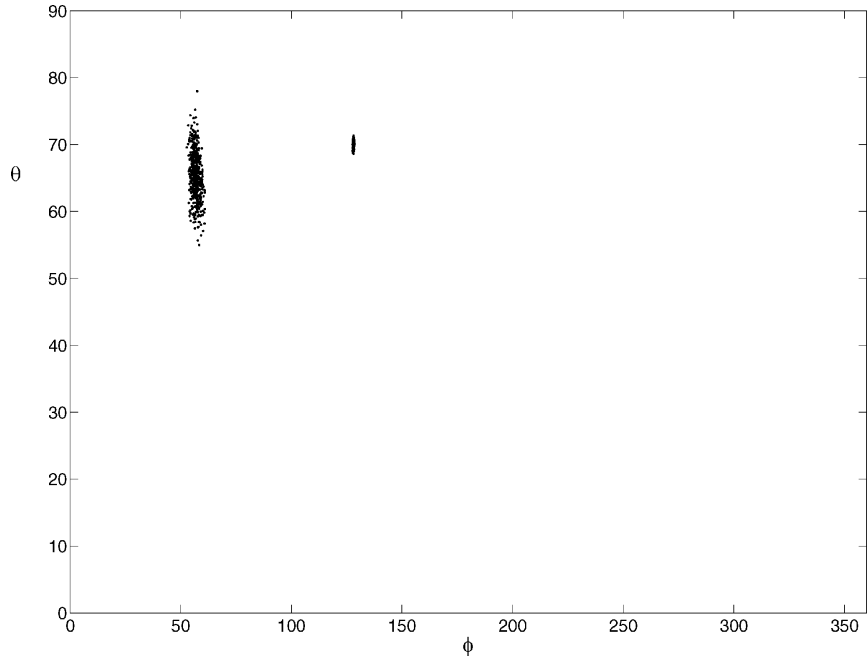

Fig. 7. The DOA estimates of 500 implementations when two sources with strongly different SNR level are impinging on the UCA.

our proposed combined estimation algorithm is close to the corresponding CRB and so we conclude that our algorithm delivers accurate estimates and it is an efficient algorithm. Notice that the traditional search of the 2-D MUSIC spectrum for high SNR values results in RMSEs which are smaller than the corresponding CRBs. This is due to the fact that the 2-D MUSIC spectrum is calculated for a discrete grid of possible DOAs and the DOAs of the three different signals are all element of this grid. So the probability to estimate the exact DOA is much larger than in the case that we study a continuous grid.

In a second example we study whether the algorithm is able to detect signals with different strength. We consider two uncorrelated sources emitting 100 bit pseudo-random bit sequences. The DOAs of the two signals are $\left(\theta_{1}, \varphi_{1}\right)=\left(70^{\circ}, 57^{\circ}\right)$ and $\left(\theta_{2}, \varphi_{2}\right)=\left(70^{\circ}, 128^{\circ}\right)$. The two signals have the same elevation angle, so the UCA receives the two signals in a similar way. The SNR level of the first signal is $5 \mathrm{~dB}$, while the second source is $20 \mathrm{~dB}$ stronger $\left(\mathrm{SNR}_{2}=25 \mathrm{~dB}\right)$. In Fig. 7 the result of 500 implementations of estimating the DOAs by the combined algorithm is shown. The two sources are well detected. In some cases the error on the estimated DOA of the weakest signal is significant. This effect reflects in the RMSEs of the signals. The RMSE of the signals are $\operatorname{RMSE}_{1}=\left(5.9^{\circ}\left[2.48^{\circ}\right], 1.5^{\circ}\left[0.62^{\circ}\right]\right)$ and $\operatorname{RMSE}_{2}=\left(0.49^{\circ}\left[0.24^{\circ}\right], 0.14^{\circ}\left[0.06^{\circ}\right]\right)$. Between brackets the corresponding CRBs are given. It is clear that the elevation angle of the weakest signal is hard to estimate. On the one hand, the low SNR level results in a large RMSE and on the other hand, a circular geometry has intrinsically smaller resolution for detecting the elevation angle. To obtain better performances for the elevation angle, more complex array configurations have to be considered.

In the last example we examine the capability of the proposed algorithm to estimate the DOAs of closely spaced signals. Subspace methods, such as MUSIC, have superb resolution properties compared to the traditional methods such as the Capon method [17]. They are called superresolution methods because they can detect two signals which are situated closer to each other than the beamwidth of the considered antenna array. 

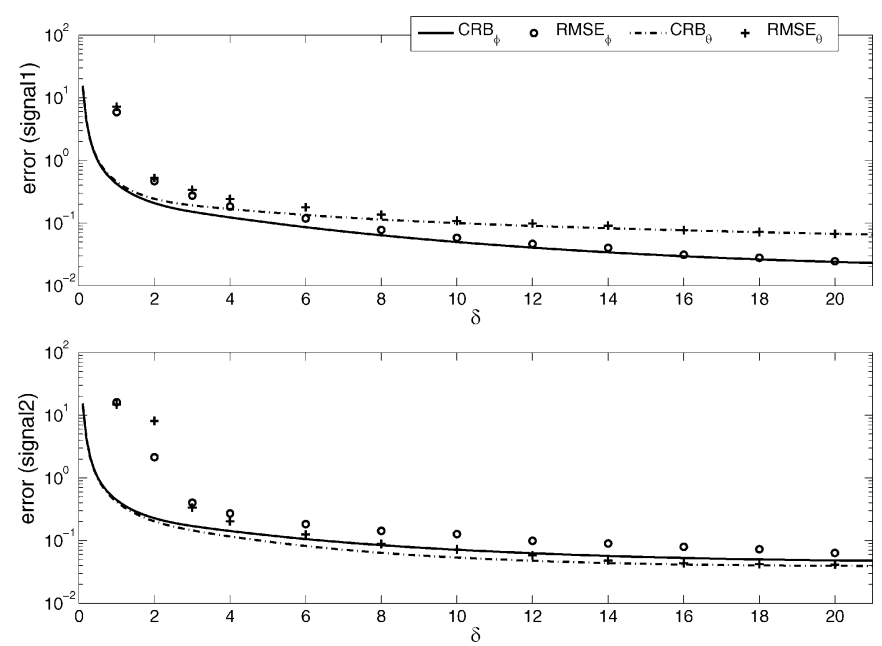

Fig. 8. RMSEs and corresponding CRBs of azimuth and elevation angles for two closely spaced sources versus source separation $\delta$.

In the first two examples we estimate the DOAs with an extremely small UCA. The radius of this array is $\lambda / 4$ and the corresponding $3 \mathrm{~dB}$ beamwidth of this array is $116^{\circ}$. The advantage of this antenna array is the limited number of antenna elements which is necessary to realize a beampattern with a constant beamwidth for every azimuth angle. In order to clearly illustrate the superresolution properties of the proposed algorithm, a UCA with a larger radius $(r=\lambda)$ is used to study whether the proposed method can handle signals which are closely spaced. The beamwidth of such a UCA is now reduced to $28^{\circ}$. Compared to the smaller UCA, a larger number of antenna elements is necessary to perform UCA-RARE. For this array 21 elements are considered and this is sufficient to properly describe all relevant phase modes of the UCA. Again an additional short-circuited dipole is placed in the center of the circle. Now we consider two uncorrelated sources emitting 100 bit pseudo-random bit sequences. The DOAs of the two signals are $\left(\theta_{1}, \varphi_{1}\right)=\left(70^{\circ}, 0^{\circ}\right)$ and $\left(\theta_{2}, \varphi_{2}\right)=\left(70^{\circ}-\delta, \delta\right)$. The DOA of the second source is varied as $\delta$ increases from $0^{\circ}$ to $20^{\circ}$. Again we assume that all signals have equal source variance

$$
E\left[s_{1}(t)^{*} s_{1}(t)\right]=E\left[s_{2}(t)^{*} s_{2}(t)\right]=P .
$$

The SNR level is referred to the first source, which has a constant DOA. The SNR level of the first source is chosen $20 \mathrm{~dB}$ $\left(\mathrm{SNR}_{1}=20 \mathrm{~dB}\right)$. The SNR level of the second source depends on $\delta$

$$
\mathrm{SNR}_{2}=\frac{g\left(70^{\circ}-\delta\right)}{g\left(70^{\circ}\right)} \mathrm{SNR}_{1}
$$

Again we estimate the DOAs with the combined algorithm and we calculate the RMSE of the estimates by averaging over an ensemble of 500 bit sequences. In Fig. 8 the RMSE of the estimates and the corresponding CRBs are shown. It is clear from the figure why subspace methods, such as MUSIC, are called superresolution methods. Although the beamwidth of the UCA is $28^{\circ}$, the UCA is still capable to estimate the DOAs of the two sources accurately when the source separation $\delta$ is $3^{\circ}$. For smaller separation angles the accuracy decreases drastically. Again we see that the proposed algorithm remains an efficient algorithm because the RMSEs are close to the corresponding CRBs.

\section{CONCLUSION}

A new method for combined azimuth and elevation DOA estimation in UCAs is presented. Special attention is devoted to the presence of mutual coupling which is a major issue in circular arrays. In a first step, an estimation of the azimuth angles is performed by the UCA-RARE algorithm which decouples the estimation of the azimuth angles from the estimation of the elevation angles. It is proved by means of a decomposition of the element manifold into phase modes that UCA-RARE is still applicable in the presence of mutual coupling. The obtained estimates of $\varphi$ by UCA-RARE reduce the DOA estimation to a 1-D search over the elevation angle $\theta$. This $1-\mathrm{D}$ search can be easily performed by extending the element manifold and applying a double Fourier expansion. The expansion of the array manifold into a double Fourier series enables us to perform a numerically efficient Root-MUSIC algorithm in elevation direction along the estimated azimuth angles. By means of some relevant examples it is proved that the proposed hybrid algorithm yields good estimates for both azimuth and elevation angles. The RMSEs tend to the CRB, which defines the ultimate accuracy of any estimation procedure. Special attention is paid in the examples to the definition of the SNR level which is not unambiguously defined in realistic antenna arrays. A challenge for further research is to incorporate a spatial smoothing scheme to deal with incoherent signals. It is far from evident to apply a spatial smoothing technique while estimating the azimuth angle as well as the elevation angle in the presence of mutual coupling.

\section{APPENDIX}

The foundation of the new algorithm in Section IV is the capability to express the element manifold as a multivariate polynomial in $z=e^{j \varphi}$ and $w=e^{j \theta}$. In [7] it is already proven that the element manifold can be expanded into a limited Fourier series of phase modes by considering a general multiport antenna, carrying a current distribution $\mathbf{C}(R, \varphi, z)$ on the surface $S$ of a cylinder with radius $R$ and height $z_{\max }$. This current distribution includes all full-wave effects, such as mutual coupling. In a same manner we prove that the element manifold can be expanded similarly into a limited Fourier series in elevation direction. Assuming that the currents are $z$-oriented $\mathbf{C}(R, \varphi, z)=C(R, \varphi, z) \mathbf{u}_{z}$, the $\theta$-oriented radiation pattern $\mathbf{F}_{\theta}=F(\theta, \varphi) \mathbf{u}_{\theta}$ is related to the current distribution by

$$
\begin{aligned}
& F(\theta, \varphi)=-j \omega \mu_{0} \sin \theta \\
& \cdot \int_{\varphi^{\prime}=0}^{2 \pi} \int_{z} C\left(R, \varphi^{\prime}, z\right) e^{j k\left(R \sin \theta \cos \left(\varphi-\varphi^{\prime}\right)+z \cos \theta\right)} \mathrm{d} \varphi^{\prime} \mathrm{d} z .
\end{aligned}
$$

To perform a Fourier transformation in elevation angle the radiation pattern is extended to the interval $\theta \in[0,2 \pi]$. Therefore, we define the modified radiation pattern

$$
\begin{aligned}
& G(\theta, \varphi) \\
& =\left\{\begin{array}{cl}
F(\theta, \varphi) & \text { when } 0<\theta<\pi \quad 0<\varphi<\pi \\
-F(2 \pi-\theta, \pi+\varphi) & \text { when } \pi<\theta<2 \pi \quad 0<\varphi<\pi
\end{array} .\right.
\end{aligned}
$$




$$
g_{n}(\varphi)=\frac{j^{n-1}}{2 j} \int_{\varphi^{\prime}} \int_{z} C\left(R, \varphi^{\prime}, z\right)\left(J_{n-1}(\alpha) e^{-j(n-1) \beta}+J_{n+1}(\alpha) e^{-j(n+1) \beta}\right) \mathrm{d} \varphi^{\prime} \mathrm{d} z .
$$

In (43) the - sign appears because $\mathbf{u}_{\theta}$ flips at the poles. The modified radiation pattern is now expanded into a Fourier series

$$
G(\theta, \varphi)=\sum_{n=-\infty}^{n=+\infty} g_{n}(\varphi) e^{j n \theta} .
$$

The $n$th phase mode is then given by

$$
\begin{aligned}
& g_{n}(\varphi)= \frac{1}{2 \pi} \int_{0}^{\pi} F(\theta, \varphi) e^{-j n \theta} \mathrm{d} \theta \\
&-\frac{1}{2 \pi} \int_{\pi}^{2 \pi} F(2 \pi-\theta, \pi+\varphi) e^{-j n \theta} \mathrm{d} \theta \\
&= \frac{1}{2 \pi} \int_{\varphi^{\prime}} \int_{z} C\left(R, \varphi^{\prime}, z\right) \mathrm{d} \varphi^{\prime} \mathrm{d} z \\
& \cdot \int_{0}^{2 \pi} \sin \theta e^{j k\left(R \sin \theta \cos \left(\varphi-\varphi^{\prime}\right)+z \cos \theta\right)} e^{-j n \theta} \mathrm{d} \theta \\
&= \frac{1}{4 j \pi} \int_{\varphi^{\prime}} \int_{z} C\left(R, \varphi^{\prime}, z\right) \mathrm{d} \varphi^{\prime} \mathrm{d} z \\
& \cdot \int_{0}^{2 \pi} e^{j \alpha \cos (\theta-\beta)}\left(e^{-j(n-1) \theta}-e^{j(n+1) \theta}\right) \mathrm{d} \theta \\
& \text { with } \quad \alpha=k \sqrt{z^{2}+R^{2} \cos ^{2}\left(\varphi-\varphi^{\prime}\right)} \\
& \text { and } \beta=\frac{R \cos \left(\varphi-\varphi^{\prime}\right)}{z} .
\end{aligned}
$$

Equation (46) can be simplified by using the Jacobi-Anger expression. This results are shown in (48) at the top of the page. It is clear that $g_{n}(\varphi)$ quickly decreases when the order $n$ increases. When the order of the Bessel function is larger than its argument, $g_{n}(\varphi)$ will become sufficiently small. This occurs when $n-1>k \sqrt{R^{2}+z_{\max }^{2}}$. The voltage induced at an antenna is directly related to the radiation pattern, so the modified array manifold can be decomposed into a limited number of phase modes with $N>k \sqrt{R^{2}+z_{\max }^{2}}+1$. This argumentation is valid for a general multiport antenna, so in particular it is valid for UCAs considered in this paper.

\section{REFERENCES}

[1] C. P. Mathews and M. D. Zoltowski, "Eigenstructure techniques for 2-D angle estimation with uniform circular arrays," IEEE Trans. Signal Processing, vol. 42, no. 9, pp. 2395-2407, Sep. 1994.

[2] H. Steyskal and J. S. Herd, "Mutual coupling compensation in small array antennas," IEEE Trans. Antennas Propag., vol. 38, pp. 1971-1975, Dec. 1990.

[3] T. Su, K. Dandekar, and H. Ling, "Simulation of mutual coupling effect in circular arrays for direction-finding applications," Microw. Opt. Technol. Lett., vol. 26, pp. 331-336, Sep. 2000.

[4] B. Friedlander and A. J. Weiss, "Direction finding in the presence of mutual coupling," IEEE Trans. Antennas Propag., vol. 39, pp. 273-284, Mar. 1991.

[5] M. Wax and J. Sheinvald, "Direction finding of coherent signals via spatial smoothing for uniform circular arrays," IEEE Trans. Antennas Propag., vol. 42, pp. 613-620, May 1994.

[6] K. Kim, T. K. Sarkar, and M. S. Palma, "Adaptive processing using a single snapshot for a nonuniformly spaced array in the presence of mutual coupling and near-field scatterers," IEEE Trans. Antenn. Propag., vol. 50, pp. 582-590, May 2002.
[7] H. Rogier and E. Bonek, "Analytical spherical-mode based compensation of mutual coupling in uniform circular arrays for direction-of-arrival estimation," Int. J. Electr. Comm., vol. 60, no. 3, pp. 179-189, Mar. 2006

[8] S. Wang, T. K. Sarkar, and S. Best, "Direction of arrival (DOA) estimation using electrically small resonant dipole antennas," in IEEE Conf. on Radar, Apr. 2006, pp. 166-173.

[9] N. Yilmazer, J. Koh, and T. K. Sarkar, "Utilization of a unitary transform for efficient computation in the matrix pencil method to find the direction of arrival," IEEE Trans. Antennas Propag., vol. 54, no. 1, pp. 175-181, Jan. 2006.

[10] R. O. Schmidt, "Multiple emitter location and signal parameter estimation," IEEE Trans. Antenn. Propag., vol. 34, pp. 276-280, Mar. 1986.

[11] H. Rogier and E. Bonek, "A model for describing mutual coupling in uniform circular arrays and its application to DOA estimation," in IEEE Antennas and Propagation Society Symp., Jun. 2004, vol. 2, pp. $1251-1254$

[12] M. Pesavento and J. F. Böhme, "Direction of arrival estimation in uniform circular arrays composed of directional elements," in Sensor Array and Multichannel Signal Processing Workshop Proc., Aug. 2002, pp. 503-507.

[13] R. Goossens and H. Rogier, "2-D direction of arrival estimation combining UCA-RARE and MUSIC for uniform circular arrays subject to mutual coupling," in Proc. Int. Conf. on Electromagnetics in Advanced Applications, Sep. 2005, pp. 791-794.

[14] M. A. Doron and E. Doron, "Wavefield modeling and array processing-Part II: Algorithms," IEEE Trans. Signal Processing, vol. 42, no. 10, pp. 2560-2570, Oct. 1994.

[15] Y. T. Lo and S. W. Lee, Antenna Handbook. New York: Van Nostrand Reinhold, 1988.

[16] P. Kildal, "Equivalent circuits of receive antennas in signal processing arrays," Microw. Opt. Technol. Lett., pp. 244-246, May 1999.

[17] J. Capon, "High-resolution frequency-wavenumber spectrum analysis," Proc. IEEE, vol. 57, pp. 1408-1418, 1969.

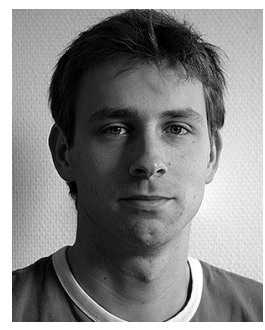

Roald Goossens was born in 1981. He received the Physical Engineering degree from Ghent University, Gent, Belgium, in 2004, where he is currently working toward the Ph.D. degree.

His current research interests are smart antenna systems for wireless networks and analysis of electromagnetic waveguides.

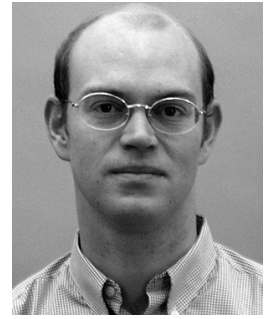

Hendrik Rogier (M'00-SM'06) was born in 1971 He received the Electrical Engineering and the Ph.D. degrees from Ghent University, Gent, Belgium, in 1994 and in 1999, respectively.

$\mathrm{He}$ is currently a Postdoctoral Research Fellow of the Fund for Scientific Research - Flanders (FWO-V), Department of Information Technology, Ghent University where he is also part-time Professor with the Department of Information Technology. From October 2003 to April 2004, he was a Visiting Scientist at the Mobile Communications Group of Vienna University of Technology. He authored and coauthored about 30 papers in international journals and about 35 contributions in conference proceedings. $\mathrm{He}$ has one patent pending. His current research interests are the analysis of electromagnetic waveguides, electromagnetic simulation techniques applied to EMC and signal integrity problems, as well as to indoor propagation and antenna design, and in smart antenna systems for wireless networks.

Dr. Rogier was twice awarded the URSI Young Scientist Award, at the 2001 URSI Symposium on Electromagnetic Theory and at the 2002 URSI General Assembly. 